Aus der I. Chirurgischen Abteilung des Allgemeinen Krankenhauses Barmbeck. (Oberarzt: Prof. Dr. Sudeck.)

\title{
Zur Ätiologie der Perthesschen Krankheit, zugleich ein Beitrag zur traumatischen Hüftgelenksluxation im Kindesalter.
}

Von Dr. Max Rehbein, Assistehzarzt.

((Mit 3 Abbildungen.)

In der Bier-Festschrift des Archivs für klinische Chirurgie hat Dölle auf die Seltenheit der traumatischen Hüftgelenks. luxation im Kindesalter hingewiesen. Er konnte unter Zuzählung zweier eigener, eingehend beschriebener Fälle aus der ganzen Weltliteratur nur 38 Fälle zusammenstellen. Wir hatten auf der hiesigen Abteilung im letzten Jahr Gelegenheit, einen hierhergehörigen Fall zu beobachten, der durch die Entwicklung der Perthesschen Krankheit auf der Basis der traumatischen Luxation besonderes Interesse bietet.

Auszug aus der Krankengeschichte:

Aufnahme am 29. XI. 1920. Pat ist heute abend bei Glatteis auf der Straße auf die r. Hüfte gefallen.

B ef und: 8. J. alter, mittrelkräftiger Knabe in gutcm Ernäh. rungszustande, innere Organe ohne Besonderheiten.

Die Geg'end der r. Hüftc ist stark geschwollen. Das r. Bein ist stark verkürzt, steht adduziert. leicht nach innen rotiert. Erheb. licher Hochstand des Trochanters. Aktive Beweglichkeit aufgehoben. Jcder Versuch einer passiven Bewegung ist außerordentlich schmerz. haft. Röntgenbefund (Prof. H a e n is ch): Die r. Hüftgelenkspfanne ist leer, der Schenkelkopf nach oben außen abgewichen. Pfanne wie Kopf sind durchaus normal gebildet. Eine Fraktur ist nirgends nachweisbar. Luxatio iliaca im r. Hüftgelenk (Abb. I).

30. XI. Reposition in leichter Narkose, die ohne jede Mühe gelingt. Ruhigstellung des $r$. Beines in Vollimannscher Schiene.

3. XII. Beginn mil Bewegungsübungen. 
8. XII. Las $r$ Bein kann in d:r ll tifte aktiv ia vollom limfange und ohne Schmorzen bewegt werden.

15. XII. Pat wird gebroilt entlasson. Altive Bewrolitheit nor. mal. Kein. Beschwerden mehr.

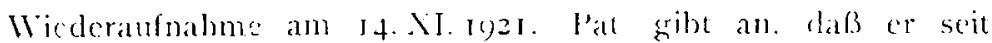
ciniger Zeit abents leichte zichend. Schmerzen aln $r$. Kne hat, daß er aut dem r. Bein, beconders math Ansirengungen, hinkt. Die

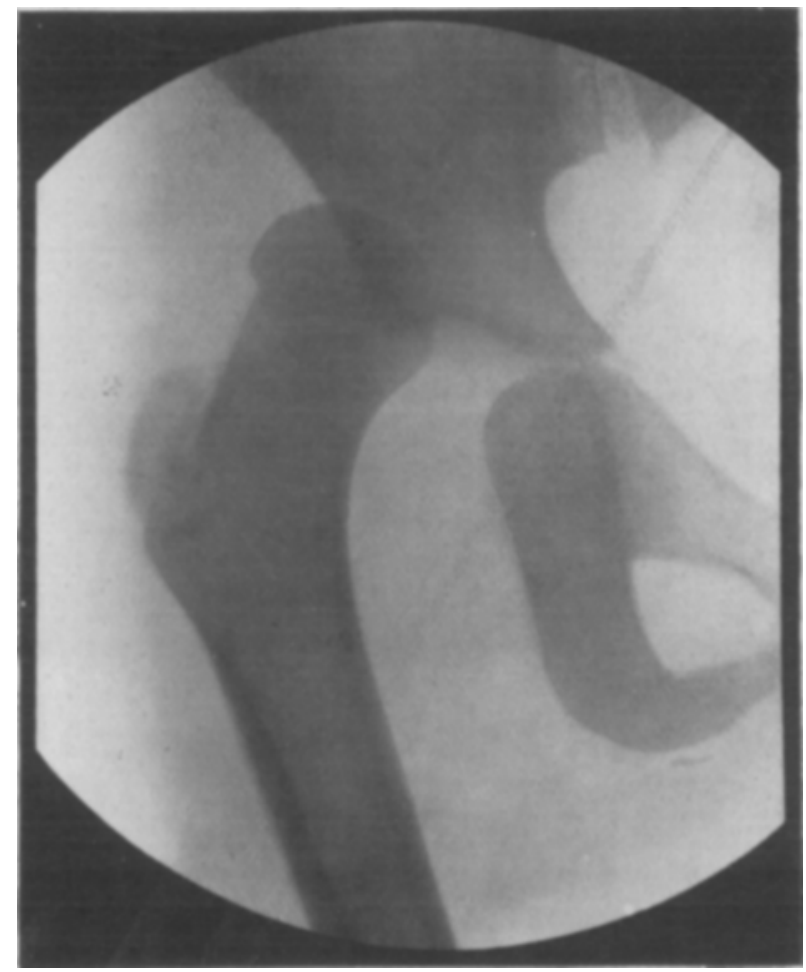

Fig. 1.

30. II, 1920. L.uxatio iliaca rechts.

Eltcrn geben ergäuzend an, daß3 der Junge schon batd nach der Entlassung aus dem Krankenhatuse angefangen habe. das r. Bein zu schonen. Da in lether \%eit sich das Hinken versehlimmert habe, hätten sie den Kasscnarzt konsultiert. Von diesem wird Pat hierher eingewiesen. In der lamilie is bisher niemals ein $\mathrm{Huftleiden}$ beobachtet worden. Pat. hat + gesunde Bruder. ist selbst immer schr

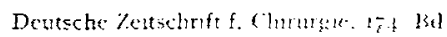


gesund gewesen. Er hat früh laufen gelernt und ist den Eltern wegen besondcrer korperlicher Gowandibeit aufgefallen

B ef und: Mittelkräftiger Knabe, in mäbigen Ërnährungszustand. Cicsunde Gesichtsfarbe. Sichtbarc Schleimhäute gut durcl-blutet. Keine Drüsen. Innere Organe gesund.

Das r. Bcin zeigt eine deutliche Muskelatrophie, im Oberschenkel ist der Lmfang $15 \mathrm{~cm}$ oberhalb des obcren Kniescheibenrandes ge-

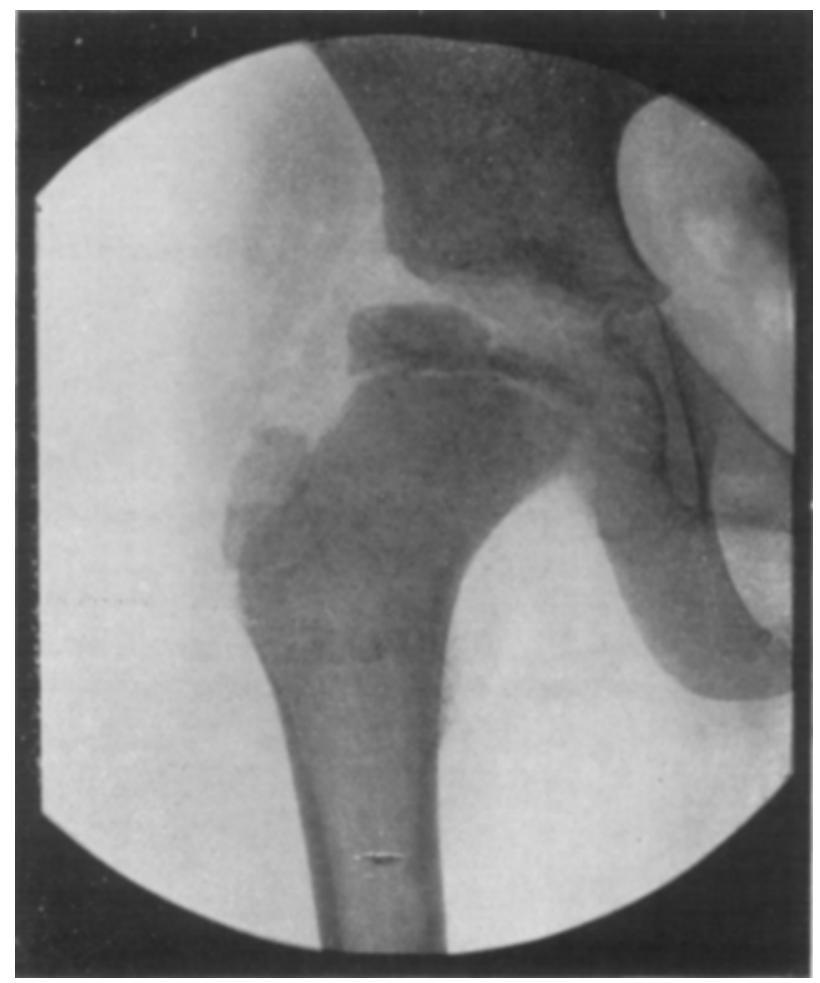

Fig. 2.

21. 11. 1921. Perthessche Krankheit rechts.

messen gegen links un $3 \mathrm{~cm}$, an der Wade um ca. $1 \mathrm{~cm}$ geringer. Die Gelenke sind mit Ausnahme des r. Hüftgelenks frei heweglich. Im r. Hüftgelenk ist aktiv die Flexion frei, die Abduktion stark eingeschränkt, die Auswärtsrotation ein wenig eingeschränkt. Passiv keine weitere Abduktion und Auswärtsrotation als aktiv möglich. Trochanter stehen beiderscits gleich hoch. Trendelenburg negativ. Keine Verkürzung des $\mathrm{r}$. Beins. Am r. Kune besteht am Ansatzpunkt 
der unteren Strecksehne an der Patella ein geringer Druckschmerz. Röntgenbcfund (Prof. Hlaenisch): Am r. Ilüftgiclenk findet sich eine erhebliche Zerstorung der Kopfkappe, dieselbe ist verbreitert, schr wesentlich abgeflacht, die Struktur stark umgebaut, stellenweise erheblich verdichtet, die Kopfkappe selbst offenbar in 2 größere Teile geteilt. Eine leichte fleckige Aufhellung geht auch in den Schenkelhals über. Der stark abgeplattete Kopf liegt in der Pfanne, etwas nach außen oben abgewichen (l'erthes).

(I. Hüftgelenk o. B. siehe Abb. 2 und 3).

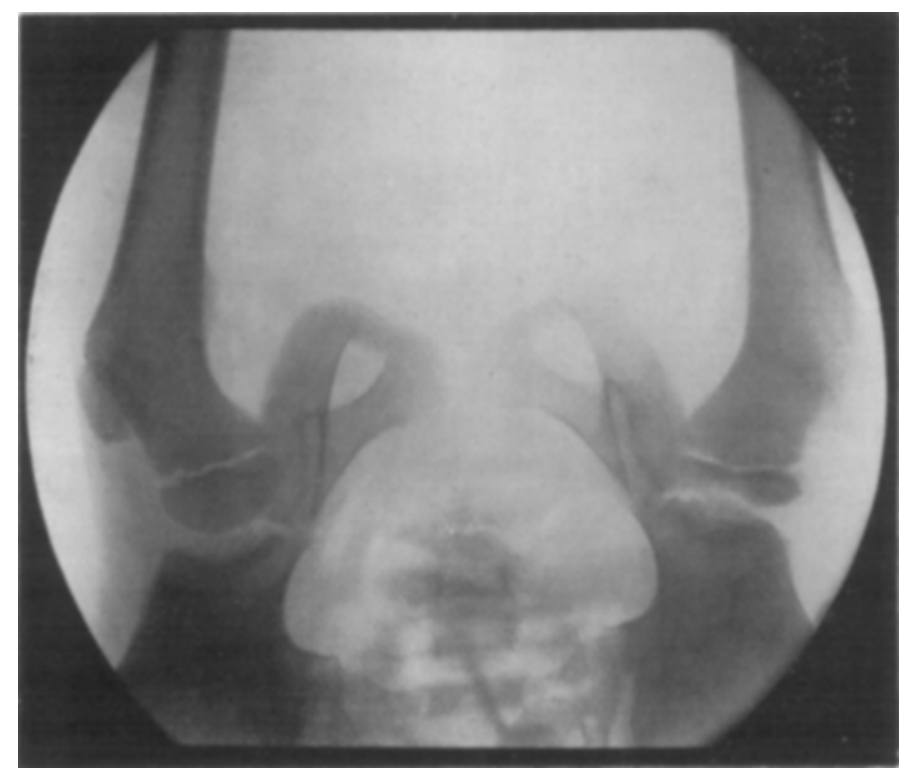

Fig. 3.

21. II. 1921. ¿'bersichtsbild. I.inkes Hüftgelenk o. B.

Epikrise: Ein $8 \mathrm{j}$. gesunder knabe aus einer liamilic, in der bisher keine Hüftgelenksleiden beobachtet worden sind. erleidet durch Sturz auf dem Glattets eine Iuxatio ilnaca femoris r. Die Röntgenaufnahme zcigt einen luxierten, völlig normal grobauten Femurkopf. Dic Reposition in Narkose gelingt leicht. Nach $2^{1}{ }^{\prime}$, Wochen wird Pat. mit funktionstüchtigem Bein entlassen. Kurz nach der Entlassung beginnt er auf dem r. Bcin zu hinken. Im Verlauf cines Jahres langsame Zunahme des Hinkens, weswegen schließlich eine Wiederaufnahme im Krankenhause erfolgt. Es finden sich nun am r. Femurkopf röntgenologisch die fur die I'erthessche Krankheit 
typischen Verinderungen. Auch klinisch die fur Perthes typischen Symptome

Die Entstehung der Osteochondritis deformans jurenilis coxae nach Reposition von kongenitaler IIüfuelenksluxation ist ein oft beobachtetes und vielbeschriebenes Bild. Auch auf der gesunden Scite hat man bei cinscitiger kongenitaler Füfugclenkisluxation nach Reposition und auch ohne Reposition dic Entstehung der Perthesschen Krankheit beobachtet (Brandes, Schwar $\mathrm{C}$ ). Einc Entstehung auf der I3asis einer traumatischen Huftgelenksluxation ist in der Litcratur bisher nicht beschrieben worden. Dic Ätologie der Perthesschen Krankheit ist noch nicht völlig seklärt. Trauma, Infektion und konstitutionelle Momente werden von den verschiedenen Autoren ursächlich angenommen. Schwarz, der die Fälle der Tübinger Klinik bearbeitet hat, ist sehr für die traumatische Cienese cingetreten. IEr glaubt, dal3 durch das Trauma cine Schädigung der Collumarterien stattindet, und daß sich die deformierenden I'rozesse sekundir auf der Basis der GefäBschädigung entwickeln. Tir sicht also das Wescn der Erkrankung in einer Ernährungsstorung des lemurkopfes. In den neueren Arbeiten ist von verschiedenen Seiten sowohl dic traumatische Genesc als auch dic Richtigkeit der Gefäßhtheoric bestritten worden. Besonders $13 \mathrm{r}$ an des, der ein guter Kenner der Osteochondritis deformans juvenilis ist, will dem Trauma in der Atiologie nur cine sehr untergeordnete Berleutung cinriumen. Er hebt nicht mit Unrecht hervor, clab die anamnestisch angeführten Traumen meist sehr geringer Natur sinsl, daß sie cigentlich gar kein Trauma für einen gesunden Menschen darstellen. Er sicht in keinen Falle den zwingenden Beweis f:ir die Möglichkeit einer rein traumatischen Genese der Perthesschen Frkrankung erbracht und glaubt, daß kongenitale Entwicklungssörungen in erster Linie als disponierende Faktoren verantwortich zu machen sind. In seiner letzten Arbeit schlickt er sich sogar $S \ddot{d}$ erlund an, der die Ansicht vertritt, daB in allen Fillen von echter Perthesscher Erkrankung niemals ein nomales oberes fiemurende bestanden hat. Brandes führt zur Stütze seiner Anschauung eincrseits die Tatsache an, daB in viclen Fällen cin familiäres Auftreten der Perthesschen Krankheit beobachtet worden ist, andererseits, dab er in zwei und $\mathrm{Sch}$ warz in cinem lalle bei cin- 
scitiger kongenitaler Luxatio coxae an der gesunden Hüfte die für den Perthes typischen deformicrenden Veränderungen auftreten gesehen hat. Gegen die Gefäßtheorie von $\mathrm{Sch}$ wa $\mathrm{r} z$ führt cr die Versuche Iselins an, der an Hunden das Ligamentum teres durchgeschnitten hat, ohne im weiteren Verlauf irgendwelchen sichtbaren Ausdruck einer Ėnährungsstörung im Femurkopf beobachten zu können. Ferner die Ansichten $\mathrm{S} \ddot{o} \mathrm{dl}$ erlunds und Severin-Nordenhof $\mathrm{s}$, dal. Epiphysenlösungen durchaus nicht das Auftreten von deformierenden Veränderungen im Schenkelkopf zu bedingen brauchen.

Nun, ich glaube. Brandes ist in seiner Ablehnung des Traumas und Betonung der Disposition zu weit gegangen. Der hier beschriebene Fall beweist cinwandfrei, daß ein Trauma allein sehr wohi genügen kann, um an einem durchaus gesunden, normal gebauten ficmurkopf die für die Perthessche Krankheit typischen Veränderungen hervorzurufen. Nllerdings ist das hier vorliegende Traumal ein stärkeres als die sonst angeführten Traumen. Söder l unds Ansicht, daß niemals ein normales obcres remurende, bestanden hat, kann nach Kenntnisnahme dieses Falles wohl nicht mehr aufrechterhalten werden. In den fällen von kongenitaler Luxation hat man das Repositionstrauma ursächlich beschuldigt. Hier licgt ein echtes Repositionstrauma ja wohl kaum ror. Der Auszug aus der Krankengeschichte zejgt, daß die Reposition ohne Mülie gelang. Also muß das Luxationstrauma als das ursächlich wirkungswolle Moment angeschen werden. Der Mechanismus der Verletzung erklärt leicht eine Schädiơnung der Ligamentum teresund Collum-Gefäße im Sinne der Schwar z schen Gefäßstheorie.

Auf diese möchte ich noch mit eirigen Worten eingchen. Schwarz gibt nach $\mathrm{L}$, exer an, daß cine Anastomose zwischen Collum- und Ligamentum teres-Gefäßen nicht bestcht. Für die Ernährung der Fpiphyse sieht er die Collumgefäße allein für wichtig an und glaubt, daß ihre Schädigung schon für clie Entstchung von Ernährungsstörungen genüge. Dieses ist nicht der Fall, wie Söderlund und Scverin-Nordenhof tganz richtig betoni haben, jenes auch nicht. Nach anatomischen feststcllunger bestehen zwischen Collum- und Ligamentum teresGefäßen selır wohl Anastomosen. Bei normalen Verhältnissen 
findet die Ernährung der Epiphyse in der Hauptsache durch die Collumarterien statt. Unter pathologischen Verhältnissen, z. B. Unterbrechung der Knorpelfuge und damit der Collumgefäße dagegen können dic Ligamentum tercs-Gefäße die Ernährung der Epiphyse ganz übernchmen. Es geht daraus hervor, daß die Ge. fäßschädigung beide Gefäßbrücken betreffen muB, um nachweisbare Ernährungsstörungen im Schenkelkopf zu verursachen. Dieses hat meines Erachtens $S \mathrm{ch}$ war $\mathrm{z}$ nicht genügend betont. Die Kenntnis dieser Verhältnisse macht uns aber auch noch ferner die Häufigkeit der Perthesschen Frkrankung bei Kindern mit kongenitaler Hüftgelenksluxation leichter verständlich. Loren 7 hat nachgewiesen, daß das Ligamentum tcres bei kongenitaler Luxatio coxae im 3. Lebensjahre bereits in 50 Proz. fehlt, da im 5. Lebensjahre sein Fehlen bereits zur Regel wird. Daß dic Schenkelköpfe solcher Kinder besonders für Ernährungsstörungen disponieren, leuchtet ohne weiteres cin. Dab bei solchen anatomischen Gefäßverhältnissen bereits cin geringes Trauma oder die Summierung von geringsten Traumen genügen, um eine Schädigung der einzigen Gefäßbrücke, die übcrdies noch durch die empfindliche Knorpelfuge verläuft, zu bewirken, erscheint auch durchaus möglich. Berücksichtigen wir ferner noch, daß in den meisten Fällen von cinseitiger kongenitaler Hüftgelenksluxation nach den Untersuchungen $\mathrm{B}$ a des auch die klinisch gesund erscheinende anderc Scite erhebliche Veränderungen aufweist, so lassen sich auch die 3 Fälle von $\mathrm{Brandes}$ und $\mathrm{Sch}$ warz mit dieser Theoric in Einklang bringen.

Auf diesem Umwege bin ich zu der Bestätigung der Ansicht Brandes von der Wichtigkeit der Disposition für die Entstchung der Perthesschen Krankheit gekommen. Daß aber auch das Trauma allein ätiologisch berücksichtigt werden muß, beweist der hier beschriebene Fall. 


\section{Literaturverzeichnis.}

I* Brandes, Deutsche Zeitschr. f. Chir. 1914, Bd. I3I, S. 232

2. - Zeitschr. f. orth. Chir. 1916, Bd. 35, S. 274.

3. - Deutsche Zeitschr. f. Chir. 1920, Bd. 155, S. 216.

4. - - Arch. f. orthop. u. Unfallchir. I920, Bd. 17, S. 527.

5. Braus, Anatomie des Menschen, Bd. 1. Springer, 1921.

6. Dólle, Bier-Festschrift. Arch. f. krlin. Chir. Igz I.

7. H off a, Orthopädische Chirurgie. Enke, I92I.

8. J o a chimsth a l, Handbuch der Orthopädie. Fischer, 1905/07.

9. Kreuter. Bruns' Beitr, z. klin. Chir I921, Bd. I22, S. 263.

10. Perthes. Deutsche Zeitschr. f. Chir., Bd. I07.

I1. - Chirurg. Kongreß I913.

12. Schwarz. Bruns' Beitr. z. klin. Chir. 1914, Bd.93, S. I. 